\title{
Lipid rafts, death receptors and CASMERs: new insights for cancer therapy
}
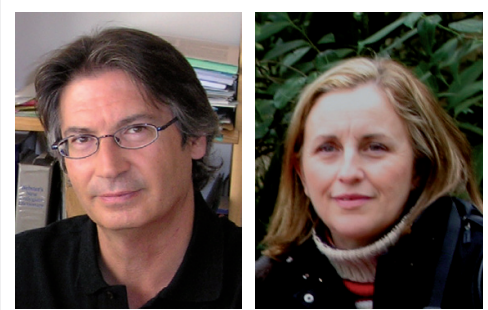

"Since the final goal in cancer therapy is to get rid of tumor cells, an appealing approach would be to set in motion the apoptotic machinery of the cancer cell to induce its own demise."

Faustino Mollinedo \& Consuelo Gajate

†Author for correspondence: Instituto de Biología Molecular y Celular del Cáncer, Centro de Investigación del Cáncer, CSIC-Universidad de Salamanca, Campus Miguel de Unamuno, E-37007 Salamanca, Spain = Tel.: +34923294806 = Fax: +34923294795 = fmollin@usal.es

Despite the tremendous efforts and budgets invested in the war on cancer, the outcome has been rather limited, to say the least, except for a number of specific cancers, such as testicular cancer, Hodgkin's lymphoma and several types of leukemia with cure rates in excess of $90 \%$. In spite of these specific successes, cancer is estimated to become the leading cause of death worldwide in 2010, according to the International Agency for Research on Cancer (IARC), a division of the WHO. The targets used for drug development have been largely nonspecific and, thus, cancer treatment has led, in several cases, to serious short- or long-term toxic side effects. The rather modest progress in the chemotherapy of cancer over the last 60 years suggests that some of the frameworks used for targeting the malignant cell are wrong, or at least not totally correct. Perception of the cancer cell as having only an uncontrolled proliferation is one such framework. Antiproliferative drugs have had only a modest impact in the clinic. This is not surprising, since many tumors have a low growth rate. Thus, there is an urgent need to search for new ways to target the tumor cell more efficiently, and to develope new drugs to overcome the rather poor return of current cancer chemotherapy.

Defects in apoptosis are a major hallmark of cancer, and contribute to drug resistance and poor outcomes in patient treatment $[1,2]$. Since the final goal in cancer therapy is to get rid of tumor cells, an appealing approach would be to set in motion the apoptotic machinery of the cancer cell to induce its own demise. Thus, direct activation of the cancer cell apoptotic arsenal constitutes an appealing therapy for the treatment of cancer that would be independent of the cell cycle and of frequently mutated tumor suppressor genes in cancer.
A remarkable trait of mammalian cells lies in the presence of death receptors at their plasma membrane. Once activated, these death receptors trigger apoptosis signaling and, hence, they can regulate cell death. This opens an interesting approach for therapeutic intervention. Cells may commit suicide if they are being encouraged to do it. By modulating the apoptosis machinery of the cancer cell, it should be feasible to enforce its own cell death. Thus, this apoptosis-targeted therapy could foster an effective destruction of the tumor. Unlike other cellular processes, proteins involved in apoptosis, such as death receptors (e.g., Fas/CD95) and adaptor proteins (e.g., FADD), lack intrinsic enzymatic activity but largely depend on protein-protein interactions to convey apoptotic signals through the assembly of major apoptotic complexes. One of these complexes is the death-inducing signaling complex [3], made up of Fas/CD95, FADD and procaspase-8, which leads to the high local concentration of procaspase- 8 molecules and its subsequent proteolytic activation by close proximity. In this regard, the concentration of apoptotic molecules in a limited region of the cell would favor the generation of apoptotic complexes and, consequently, apoptosis.

$$
\begin{aligned}
& \text { "Defects in apoptosis are a major } \\
& \text { hallmark of cancer, and contribute to } \\
& \text { drug resistance and poor outcomes in } \\
& \text { patient treatment." }
\end{aligned}
$$

\section{Lipid rafts \& death receptor signaling}

Recent evidence shows that sphingolipid- and cholesterol-rich membrane microdomains, named 'lipid rafts', serve as platforms for the recruitment and concentration of apoptotic signaling molecules at the plasma membrane [4-7]. In 2001, we 


\section{Editorial Mollinedo \& Gajate}

found that the antitumor alkyl-lysophospholipid analog edelfosine (1-O-octadecyl-2- $O$-methylrac-glycero-3-phosphocholine; ET-18- $\mathrm{OCH}_{3}$ ) induced tumor cell killing through co-clustering of Fas/CD95 death receptor in lipid rafts [8]. Subsequent studies showed that edelfosine induced translocation of additional apoptotic downstream signaling molecules into membrane rafts, including FADD, procaspase-8, procaspase-10, BH3-interacting domain death agonist (Bid), JNK and apoptosome constituents [4,7,9-11]. The co-clustering of Fas/CD95 death receptor and lipid rafts was independent of its cognate natural ligand and could be modulated pharmacologically [4]. This latter aspect opens up a promising approach in cancer treatment, as the recruitment of apoptotic molecules in rafts may become an emerging target for therapeutic intervention. In fact, following the pioneering work with edelfosine [8], a growing number of antitumor agents are being reported to promote apoptosis of cancer cells through co-clustering of
Fas/CD95 death receptor and lipid rafts, including aplidin, cisplatin, perifosine, resveratrol and rituximab [9,12-16].

Additional death receptors, particularly TNFrelated apoptosis-inducing ligand (TRAIL) receptors, have also been reported to be translocated to lipid rafts during the induction of apoptosis $[9,12,15,17]$. The co-aggregation of distinct death receptors and rafts sensitizes cancer cells to their cognate ligands $[9,17]$. In this context, TRAIL shows an encouraging antitumor activity and is currently in clinical trials [18-20]; treatment of multiple myeloma cells with edelfosine and perifosine, which promote death receptorenriched raft clusters [9], sensitizes these cancer cells to the action of TRAIL $[9,21]$.

\section{CASMER \& cancer therapy}

Since accumulating evidence indicates that the clustering of apoptotic molecules in lipid rafts is a general process involved in apoptosis, we have coined the word 'CASMER' as

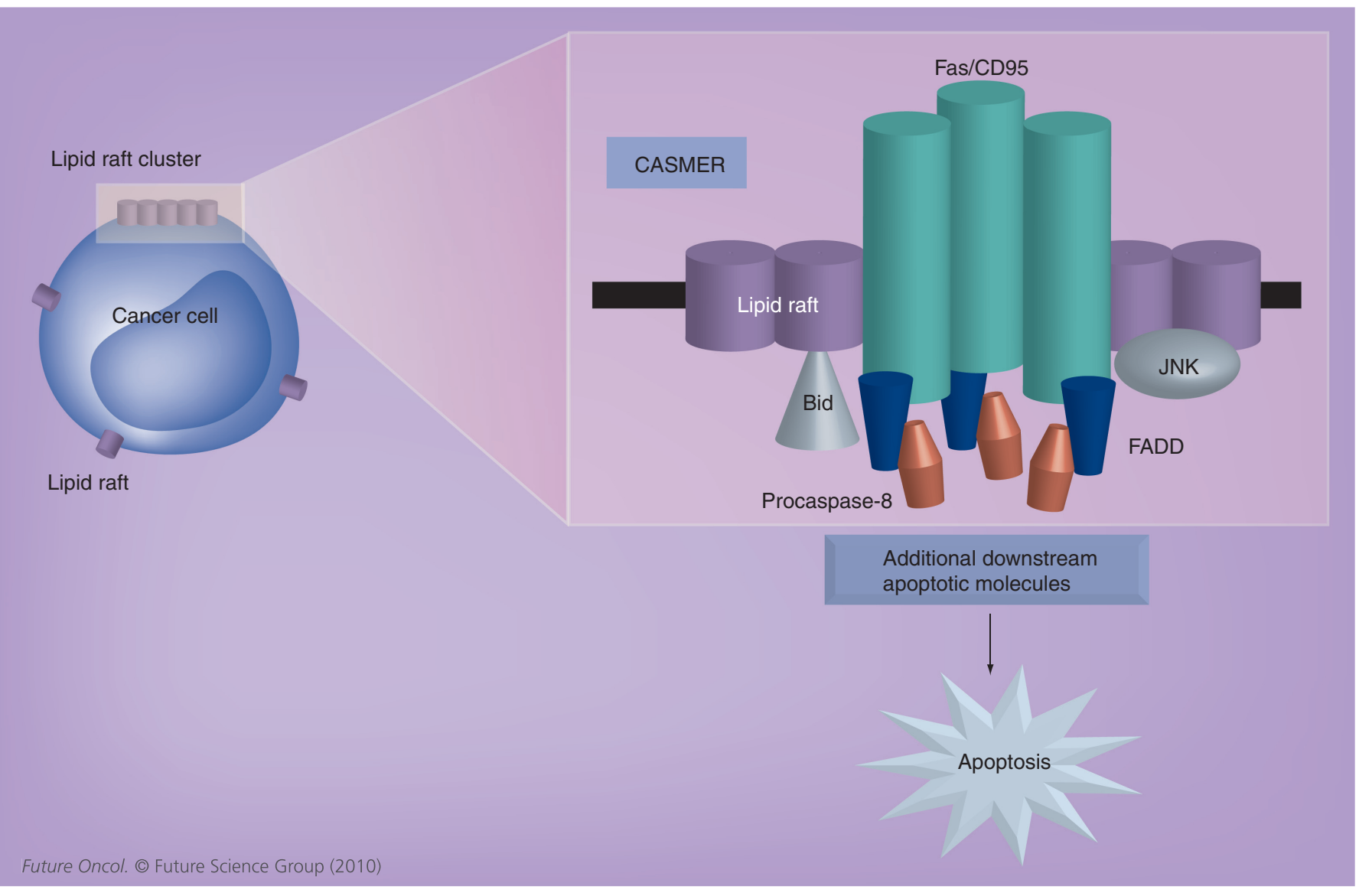

Figure 1. CASMER formation in cancer treatment. A number of antitumor agents (discussed in the article) induce clusters of plasma membrane lipid rafts, which are enriched in a variable number of death receptors and downstream apoptotic signaling molecules (i.e., CASMER). This recruitment of death receptors and apoptotic molecules in raft clusters, leading to the formation of CASMERs, promotes the generation of apoptosis-prone platforms or membrane compartments that eventually results in the death of the cancer cell by apoptosis.

Bid: BH3-interacting domain death agonist; CASMER: Cluster of apoptotic signaling molecule-enriched rafts. 
an acronym of 'cluster of apoptotic signaling molecule-enriched rafts' $[4,11,12]$ to refer to this recruitment of death receptors together with downstream apoptotic signaling molecules in aggregated rafts (FIGURE 1). CASMER represents a novel lipid-raft-based supramolecular entity that seems to play a critical role in the regulation of apoptosis, acting as a death-promoting scaffold where death receptors and downstream signaling molecules are brought together, thus facilitating protein-protein interactions and the transmission of apoptotic signals.

\section{"... CASMER may form an Achilles' heel for cancer cells."}

The ability to generate CASMERs, as well as their protein composition, depends on both the cell phenotype and the triggering stimulus $[4,9,12,15]$. Cholesterol is a critical constituent of lipid rafts, and its displacement disrupts CASMERs [4,7-9]. This is of particular interest, as cancer cells have been reported to be enriched in cholesterol [22,23]; and to display higher levels of cholesterol-rich lipid rafts than their normal counterparts [24]. On these grounds, it could be envisaged that cancer cells might potentially be prone to form CASMERs, thus becoming a convenient therapeutic target. In this way, CASMER may form an Achilles' heel for cancer cells. The recruitment of part of the apoptotic machinery of tumor cells in rafts, forming the CASMERs, from which apoptotic signals are launched, leading eventually to cell death, makes cancer cells more vulnerable.

Since CASMER formation directly triggers the apoptotic machinery, the potential for CASMER-based therapies seems very promising in the treatment of cancer and other pathologies where the stimulation of apoptosis is defective. We are entering an exciting area in which novel frameworks in apoptosis-targeted therapy may improve efficiency in cancer treatment.
CASMER-mediated direct activation of apoptosis opens a new avenue in cancer therapy, which would be independent of tumor suppressor genes (e.g., $p 53$ ) that are frequently mutated in cancer. The concept of CASMER is also related to the notion of subcellular compartmentalization of biological processes. CASMERs act as scaffolds for the recruitment of apoptotic molecules, and this highlights the existence of platforms or membrane compartments, where proteins specialized in apoptosis are brought together to facilitate their physical interaction in order to prompt a potent apoptotic response.

The mechanism of action of the phospholipid ether edelfosine can be considered as the paradigm for this new CASMER-mediated anticancer therapeutic approach. The elucidation of how this drug promotes cancer cell killing through CASMER formation should aid the design of new and more effective anticancer drugs.

\section{Financial \& competing interests disclosure \\ Work from the authors' laboratories, described in this study, was supported by grants from Ministerio de Ciencia e Innovación (SAF2005-04293, SAF2008- 02251, and and RD06/0020/1037 from Red Temática de Investigación Cooperativa en Cáncer, Instituto de Salud Carlos III, cofunded by the Fondo Europeo de Desarrollo Regional of the European Union), Fondo de Investigación Sanitaria - Instituto de Salud Carlos III and European Commission (FIS-FEDER 06/0813 and PS09/01915), Junta de Castilla y León (GR15- Experimental Therapeutics and Translational Oncology Program, and Biomedicine Project 2009). Consuelo Gajate is supported by the Ramón y Cajal Program from the Ministerio de Ciencia e Innovación of Spain. The authors have no other relevant affilia- tions or financial involvement with any organization or entity with a financial interest in or financial conflict with the subject matter or materials discussed in the manuscript apart from those disclosed. \\ No writing assistance was utilized in the production of this manuscript.}

\section{Bibliography}

1. Hanahan D, Weinberg RA: The hallmarks of cancer. Cell 100, 57-70 (2000).

2. Gajate C, Mollinedo F: Biological activities, mechanisms of action and biomedical prospect of the antitumor ether phospholipid ET-18- $\mathrm{OCH}_{3}$ (edelfosine), a proapoptotic agent in tumor cells. Curr. Drug Metab. 3, 491-525 (2002)

3. Peter ME, Krammer PH:

The CD95(APO-1/Fas) DISC and beyond. Cell Death Differ. 10, 26-35 (2003).
Gajate C, del Canto-Janez E, Acuna AU et al. Intracellular triggering of Fas aggregation and recruitment of apoptotic molecules into Fas-enriched rafts in selective tumor cell apoptosis. J. Exp. Med. 200, 353-365 (2004).

5. Mollinedo F, Gajate C: FasL-independent activation of Fas. In: Fas Signaling. Wajant $\mathrm{H}$ (Ed.). Landes Bioscience and Springer Science, TX, USA, Chapter 2, 13-27 (2006).

6. Mollinedo F, Gajate C: Fas/CD95 death receptor and lipid rafts: new targets for apoptosis-directed cancer therapy. Drug Resist. Updat. 9, 51-73 (2006).
Gajate C, Gonzalez-Camacho F, Mollinedo F: Involvement of raft aggregates enriched in Fas/CD95 death-inducing signaling complex in the antileukemic action of edelfosine in Jurkat cells. PLoS ONE 4, E5044 (2009).

8. Gajate C, Mollinedo F: The antitumor ether lipid ET-18- $\mathrm{OCH}_{3}$ induces apoptosis through translocation and capping of Fas/CD95 into membrane rafts in human leukemic cells. Blood 98, 3860-3863 (2001). 
9. Gajate C, Mollinedo F: Edelfosine and perifosine induce selective apoptosis in multiple myeloma by recruitment of death receptors and downstream signaling molecules into lipid rafts. Blood 109, 711-719 (2007).

10. Nieto-Miguel T, Gajate C, Gonzalez-Camacho F, Mollinedo F: Proapoptotic role of $\mathrm{Hsp} 90$ by its interaction with c-Jun $\mathrm{N}$-terminal kinase in lipid rafts in edelfosine-mediated antileukemic therapy. Oncogene 27, 1779-1787 (2008).

11. Gajate C, Gonzalez-Camacho F, Mollinedo F: Lipid raft connection between extrinsic and intrinsic apoptotic pathways. Biochem. Biophys. Res. Commun. 380, 780-784 (2009).

12. Gajate C, Mollinedo F: Cytoskeleton-mediated death receptor and ligand concentration in lipid rafts forms apoptosis-promoting clusters in cancer chemotherapy. J. Biol. Chem. 280, 11641-11647 (2005).

13. Lacour S, Hammann A, Grazide S et al: Cisplatin-induced CD95 redistribution into membrane lipid rafts of HT29 human colon cancer cells. Cancer Res. 64, 3593-3598 (2004).

14. Delmas D, Rebe C, Lacour S et al.: Resveratrolinduced apoptosis is associated with Fas redistribution in the rafts and the formation of a death-inducing signaling complex in colon cancer cells. J. Biol. Chem. 278, 41482-41490 (2003).

15. Reis-Sobreiro M, Gajate C, Mollinedo F: Involvement of mitochondria and recruitment of Fas/CD95 signaling in lipid rafts in resveratrol-mediated antimyeloma and antileukemia actions. Oncogene 28, 3221-3234 (2009).

16. Stel AJ, Ten Cate B, Jacobs S et al.: Fas receptor clustering and involvement of the death receptor pathway in rituximabmediated apoptosis with concomitant sensitization of lymphoma B cells to Fas-induced apoptosis. J. Immunol. 178, 2287-2295 (2007).

17. Delmas D, Rebe C, Micheau O et al.: Redistribution of CD95, DR4 and DR5 in rafts accounts for the synergistic toxicity of resveratrol and death receptor ligands in colon carcinoma cells. Oncogene 23, 8979-8986 (2004).

18. Buchsbaum DJ, Forero-Torres A, LoBuglio AF: TRAIL-receptor antibodies as a potential cancer treatment. Future Oncol. 3(4), 405-409 (2007).

19. Wang S: The promise of cancer therapeutics targeting the TNF-related apoptosis-inducing ligand and TRAIL receptor pathway. Oncogene 27, 6207-6215 (2008).

20. Kruyt FA: TRAIL and cancer therapy. Cancer Lett. 263, 14-25 (2008).

21. David E, Sinha R, Chen J, Sun SY, Kaufman JL, Lonial S: Perifosine synergistically enhances TRAIL-induced myeloma cell apoptosis via up-regulation of death receptors. Clin. Cancer Res. 14, 5090-5098 (2008).
22. Freeman MR, Solomon KR: Cholesterol and prostate cancer. J. Cell. Biochem. 91, 54-69 (2004).

23. Tosi MR, Tugnoli V: Cholesteryl esters in malignancy. Clin. Chim. Acta 359, 27-45 (2005).

24. Li YC, Park MJ, Ye SK, Kim CW, Kim YN: Elevated levels of cholesterol-rich lipid rafts in cancer cells are correlated with apoptosis sensitivity induced by cholesterol-depleting agents. Am. J. Pathol. 168, 1107-1118 (2006).

\section{Affiliations}

- Faustino Mollinedo, PhD Instituto de Biología Molecular y Celular del Cáncer, Centro de Investigación del Cáncer, CSIC-Universidad de Salamanca, Campus Miguel de Unamuno, E-37007

Salamanca, Spain

Tel.: +34923294806

Fax: +34923294795

fmollin@usal.es

- Consuelo Gajate, PhD

Unidad de Investigación, Hospital

Universitario de Salamanca, Campus

Miguel de Unamuno, E-37007

Salamanca, Spain

Tel.: +34923294806

Fax: +34923294795

cgajate@usal.es 